Original Article

\title{
ROOT CEM ENTUM INVASION AND ADHESION BY ENTEROCOCCUS FAECALIS CONFOCAL ANALYSIS.
}

\author{
Rahul Halkai ${ }^{1}$, Mithra N. Hegde ${ }^{2}$, Kiran Halkai ${ }^{3}$ \\ ${ }^{1}$ Ph.D. Student, ${ }^{2}$ Senior Professor \& Head of the Department, Department of Conservative and Endodontics, A.B. Shetty \\ M emorial Institute of Dental Science, Deralakatte, M angalore - 575 018, Karnataka, India. \\ ${ }^{3}$ Sr. Lecture, Department of Conservative and Endodontics, Sn Hke'S Dental college, GULBARGA. \\ Correspondence: \\ Rahul Halkai \\ Ph.D. Student, Department of Conservative and Endodontics, A.B. Shetty Memorial Institute of Dental Science, \\ Deralakatte, Mangalore - 575 018, Karnataka, India. \\ Mobile : +919481636661 E-mail : drrahulendo@yahoo.co.in
}

\begin{abstract}
:
Aims to know ability of Enterococcusfaecalis invasion and adhesion into root cementum.

Methodology Forty single rooted human intact teeth were selected, group I with 20 teeth as control group with no access opening and apical seal with varnish. Group II with 20 teeth, after access opening and canal debridement, all the samples were subjected for gamma sterilization to ensure complete absence of microorganisms, and then exposed to Enterococcus faecalis broth, in group II broth is placed with the help of micro pipette into root canal and at the same time apical $1 / 3$ of tooth were immersed into broth with both groups for 8 weeks. Biomechanical preparation, obturation and coronal sealing done using GIC with group II, followed by apical $1 / 3$ immersed in the enterococcus broth for 8 weeks, examination under confocal laser scanning microscope after splitting the teeth samples into two halfs buccolingually.
\end{abstract}

Results: This study shows only adhesion of Enterococcus faecalis to root cementum. Conclusion Adhesion of Enterococcus faecalis to root cementum may be the possible reason for persisting infection after root canal treatment.

Keywords: Enterocoocus faecalis, persisting infection, root cementum, confocal laser scanning microscope.

Introduction:

Bacteria are located in inaccessible areas such as complicated root canal anatomy and dentinal tubules, and it is difficult to deliver antibacterial agents to these locations ${ }^{1}$. Bacteria may survive and recolonize the root canal space whenever there is opportunity, and this may become a focal source for persistent infection, it is difficult to render the root canal free from bacteria. Although the mechanism of bacterial invasion is not completely understood, bacterial adhesion to dentinal tubule walls (TWs) is a logical early step in Access this article online the process. Collagen is widely considered to be the primary substrate for specific binding of $E$. faecalis to dentine, and the collagen binding protein of $\mathrm{E}$. faecalis (Ace) and a serine protease
(Spr) have been proposed to play significant roles in binding to the root canal wall ${ }^{2}$. Ace also promotes $\mathrm{E}$. Faecalis binding to collagen type $\mathrm{I}^{3,4}$, and in vitro ace gene expression at 37 _ $C$ was enhanced in the presence of collagen ${ }^{5}$. However, the interaction of $E$. faecalis specifically with dentinal tubules has never been investigated.

The bacteria's ability to penetrate into dentin can be determined through microbiological analyses by the number of colony forming units (CFUs) ${ }^{6}$ the number of tubules infected in the histologic samples ${ }^{7}$ or the presence of bacteria in the root canal wall ${ }^{8}$. Each method has advantage and disadvantage. Microbiological sampling techniques can estimate the number of colony forming units but however this method does not give clear information about the spatial distribution of bacteria. Histological section shows the distribution of bacteria in infected dentin but not about viability of bacteria. 
Transmission microscope provide high resolution images of bacteria but technique spends time and requires multiple steps ${ }^{9}$. Confocal laser scanning microscope allows getting series of optical sections as thin as $0.3 \mathrm{um}$ and vital staining technique to determine the viability profile ${ }^{10}$. The aim of this study is to know ability of Enterococcus faecalis invasion and adhesion to root cementum.

\section{Methodology:}

The present in-vitro study was conducted in the Department of Conservative Dentistry and Endodontics, $A B$ Shetty Memorial Institute of Dental Sciences Mangalore. Central Research Laboratories AB Shetty Memorial Institute of Dental sciences. Teeth sterilization (gamma irradiation) done at Microtol, Bangalore. Data collection done using inverted confocal laser scanning microscope ${ }^{9}$ (ZEISS LSM 510 META. GmbH, Mannheim, Germany) at Indian Institute of Sciences (IISc) Bangalore. Materials used for study (fig 1) a) Effendroph b) Micropipette c) Micropipette tube d) Teeth sample in sterilization pouch. E) Trypton Soyabean Agar broth

\section{Selection of Samples:}

Forty human single rooted teeth recently extracted for orthodontic reasons were collected for the study. After extraction the teeth were stored in chlorhexidine solution, until collection of all teeth.

\section{Inclusion Criteria :}

Single rooted caries free teeth, examined under $20 x$ magnification under a microscope to rule out any cracks, caries, fractures or craze lines and radiographed to determine the presence of a single canal were included for the present study.

\section{Exclusion Criteria}

Teeth that had already undergone root canal treatment or teeth with more than one canal, immature root apices, teeth with root caries, restorations, fracture or craze lines, thin curved roots, calcified canals were excluded from the study.

\section{Methodology:}

The teeth were cleaned off soft tissue, calculus and stains with the help of sharp hand scalers and thoroughly washed under running tap water to remove any remaining tissue remnants sticking to the tooth surface and were stored in normal saline solution at room temperature until further use.

All the forty specimens were randomly divided in two experimental groups as follows and coding of samples done (fig2).

Group 1( $n=20)$ : (control group): Intact teeth with no access cavity preparation and sealing of the root apex done using varnish.

Group 2 ( $n=20)$ : Access cavity preparation done.

\section{Procedure:}

All the samples in group 1 (controlled group), no access opening was done and from the apex to apical 1 to $2 \mathrm{~mm}$ of the teeth sealed with two coats of varnish followed by gamma irradiation of all the samples to eradicate bacteria which in case were existing before. All the specimens in Group 2, access opening, canal debridement was done and subjected to gamma irradiation, followed by culturing with E.faecalis within root canal and apical one third of teeth submerged in the broth with group I and group II samples and subjected for culturing for 8 weeks.

Culturing Procedure: Enterococcus Faecalis streptomycin resistant strains were cultured in Trypton Soyabean Agar broth. Broth is prepared by mixing the 1.8 grams powder in $60 \mathrm{ml}$ of distilled water. The prepared broth is sterilized in Autoclave, after that the strains are inoculated in the broth (fig 3) and placed in an Incubator for the bacteria to grow at $37^{\circ} \mathrm{C}$ (fig 4) for 24-48hrs. For confirmation of the bacteria Gram staining was done (fig 5). The cultured broth was inoculated within root canal of the teeth samples with a micropipette, and also apical one third submerged in the broth (fig 6). The whole process was refreshed every alternative two days for a period of 8weeks. 


\section{Biomechanical Preparation \& Obturation}

After 8 weeks of culturing all the specimens of group 2 were subjected to biomechanical preparation followed by obturation up to the working length. Working length was determined by using Root ZX II (J.M orita, Japan). The teeth were instrumented using Protaper $\mathrm{Ni}$-Ti rotary instrument system in a contra angle gear reduction hand piece ( $X$ Smart Dentsply), finally obturated with gutta preach single cone using $\mathrm{AH}$ plus sealer.

Culture and Observation: After biomechanical preparation $\&$ obturation all the teeth specimens were again immersed into E.Feacalis broth for 8 weeks. After incubation period all the samples were washed using $1 \mathrm{ml}$ phosphate buffered saline (PBS) to remove nonadherent bacteria ${ }^{11}$. A vertical groove is made on bucco lingual surface starting from occlusal surface to apical tip using tapered fissure diamond point, than with the help of chisel splitted in two half's segments (fig 7). Each half of the spitted segment was observed for the type of cemento dentinal junction under spectrophotometery microscope. All the teeth samples were observed under inverted confocal laser scanning microscope ( ZEISS LSM 510 META. GmbH, Mannheim, Germany). After coding the samples, teeth were stained with $50 \mu \mathrm{L}$ fluorescein diacetate (FDA sigma, st Louis, M O) and $50 \mu \mathrm{L}$ of propidium iodide (PI, Sigma). FDA is nonfluorescent cell permeable dye that is converted to fluorescein (green) by intracellular esterases produced by metabolically active microorganism ${ }^{10}$. PI is a fluorescent molecule impermeable to the cellular membrane and generally excluded from viable cell thus live bacterial cells are fluorescent green, whereas dead bacteria with damaged membranes are fluorescent red ${ }^{12}$.

\section{CLSM Analysis:}

The group I and II samples were examined on inverted CLSM beyond Cemento Dentinal Junction (CDJ) for the presence of $\mathrm{E}$. Faecalis. Data was subjected to appropriate statistical analysis.

\section{Results:}

Group I (fig 8) samples showed absence of Enterococcus faecalis in root cementum on other hand group II FDA/PI staining technique showed red and green colour coccoidal structure adhere to root cementum under inverted confocal microscope (fig 9). Separate picture of Dead (red) and live (green) staining (Fig 10) Results were subjected to statistical analysis using $Z$ test. $Z$ value (table 1 ) is 17.89 (?1.96 for $p=0.05$ ) this shows significant presence of Enterococcus Faecalis in group II.

\section{Discussion :}

In this study E Faecalis was chosen as the test organism because Enterococcus faecalis found to penetrate deep into dentinal tubules in vitro ${ }^{13}$, and one among the micro organisms found in reinfection or persistent lesions ${ }^{14}$. Enterococcus faecalis produces protein during stress like Ace and serine protease (spr) protein ${ }^{15}$, which in turn helps to adhere type I \& IV collagen present in root cementum ${ }^{3}$.

The adhesion of E Faecalis to type I and IV collagen is the basis of my study because, root cementum at apical one third of root is made of cellular cementum and majorly of type IV collagen.

Cementocytes are interconnected by canniculli through which nutrients from periradicular region reaches to cementocytes deep inside the cementum which help to survive. Canniculli may be the potential gateway for enterococcus faecalis to invade into root cementum in apical one third of the teeth. Other factors facilitating the presence of Enterococcus faecalis in obturated root canals with persistent lesions include, it can survive in high $\mathrm{pH}^{16}$ long starvation periods without nutrients, and in presence of serum it has the ability to recover ${ }^{17}$.

Bacteria encounters with variety of stressful conditions in the root canal, such as nutrient deficiency, other bacterial toxins, and intra canal medicaments, these conditions may regulate bacterial adhesin expression. Addition to it, when ever serum leakage into the root canal, inducing the expression of Aggregation substance (AS) and other carbohydrate moieties, thereby helping bacteria to adhere, even alkaline $\mathrm{pH}$ obtained by calcium hydroxide at the dentinal zone is in effective due to deeper penetration of 
Enterococcus faecalis ${ }^{19}$. Other mechanisms of survival may be through Lipoteichoic acids (LTA), which resist the bacterium against many lethal conditions ${ }^{19}$.

E. faecalis prevents the growth of other bacteria with cytolysin, AS-48 (Aggregation substance), and bacteriocins. Erythrocytes are the target cells of cytolysin ${ }^{20}$ also PM Ns and macrophages and a broad range of Gram-positive but not Gram-negative organisms ${ }^{21}$. The bacteriocin effect of cytolysin of E. faecalis helps colonization of the Gramnegatives, there could be a shift of bacterial flora related with periodontal disease ${ }^{22}$, the latter factors is non pathogenic in humans. Along with cytolysin, they facilitate the dominance of $E$. faecalis in a mixed infection and serve as means to obtain ecological advantages which can result in disease in man. The root canal hardly contains any nutrient-rich medium, but when required $\mathrm{E}$. faecalis may derive the energy it needs from the hyaluronan present in the dentin by breaking down hyaluronidase or $\mathrm{E}$. faecalis may also feed on fluid present in the dentinal tubules. So it seems that even, well obturated fluid tight seal does effect the survival of Enterococcus faecalis because it can generate energy to survive. The foci of infection is $E$ Faecalis present deep inside the dentin or cementum that cannot be reached by host defence cells such as PNM, lymphocytes and macrophages etc, E faecalis elicits permanent provocative effect on the host defence cell mechanism which in turn damages periradicular region.

We used gamma irradiation to sterilize the teeth because it does not alter collagen characteristics which is very important for E Faecalis to adhere to collagen, other methods of sterilization of teeth samples are by autoclave, hot air oven etc. Disadvantages of autoclave is it collapses the collagen strands and use of hot air oven makes teeth dehydrated and more brittle.

Previously most of the methods like fluorescent probes used failed to detect viability of bacterial and also spatial distribution ${ }^{23}$, bacterial viability could be checked by using conventional fluorescence microscopy but it has it disadvantage of decalcification the teeth samples, creating artificial condition, high background haze makes difficult to distinguish between individual cells ${ }^{5}$. In this context, better methodologies for the identification of bacterial viability in cementum are needed. The inverted CLSM analysis has the advantage over the conventional fluorescence microscopy to visualize bacteria. In fact our research confirms ability of $E$ Faecalis to infect root cementum and substantial improvements that show the vitality of the bacteria in root cementum. The visualisation of live and dead bacteria in root cementum using confocal microscopy, helps to determine the presence and adhesion of E.faecalis in the root cementum at the cellular level.

\section{Conclusion:}

Bacterial adhesion to dentinal tubule walls (TWs) is a logical early step in the process. Collagen is widely considered to be the primary substrate for specific binding of $E$. faecalis to cementum, and the collagen binding protein of $\mathrm{E}$. faecalis (Ace) and a serine protease (Spr) have been proposed to play significant roles in binding to the root canal wall. Ace also promotes $\mathrm{E}$. faecalis binding to collagen type I and IV ${ }^{16}$ and in vitro ace gene expression at $37^{\circ} \mathrm{C}$ was enhanced in the presence of collagen ${ }^{5}$. There is $40 \%$ available collagen present in root cementum, collagen presence in root cementum might help enterococcus faecalis to inhabit in cementum, and might be possible region for treatment failure or in ability to control infection at root apex.

Tables 1: Data calculated applying Z test.

\begin{tabular}{|l|c|c|}
\hline & $\begin{array}{c}\text { Group I } \\
\text { (control group) }\end{array}$ & $\begin{array}{c}\text { Group II } \\
\text { (apical 1/ 3 exposed to 5.5 ph) }\end{array}$ \\
\hline $\begin{array}{l}\text { Proportion of } \\
\text { presence of } \\
\text { Enterococcus } \\
\text { Faecalis in the } \\
\text { samples }\end{array}$ & 0 & \\
\hline$Z$ value & 17.89 & \\
\hline
\end{tabular}




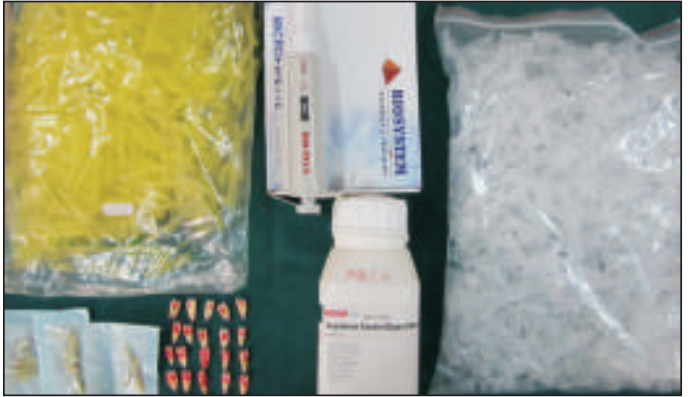

Fig 1: All the material used in the study, a) Effendroph b) Micropipette c) Micropipette tube d) teeth sample in sterilization pouch. E) Trypton Soyabean Agar broth

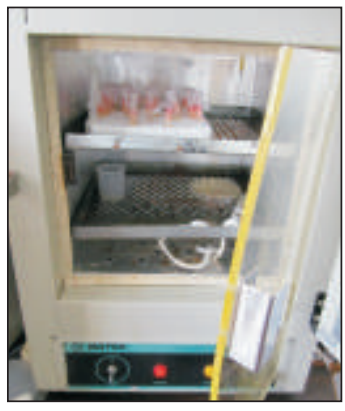

Fig 4: Gram staining shows gram +ve Cocci agganged in chains and in clusters.

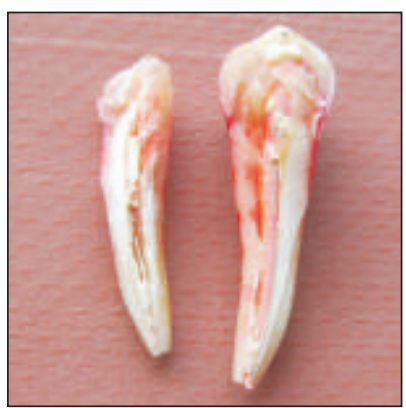

Fig 7: teeth sample spited into two half's.

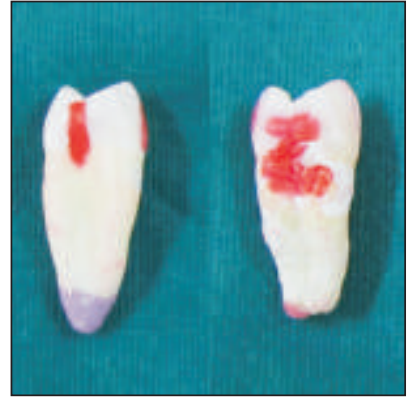

Fig 2: Coding of teeth samples group I(1) \&II(2).

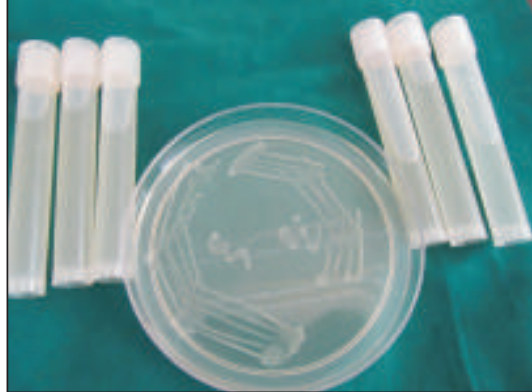

Fig 3: Enteroccocus Faecalis culture and inoculation of Enterococcus Faecalis culture in broth.

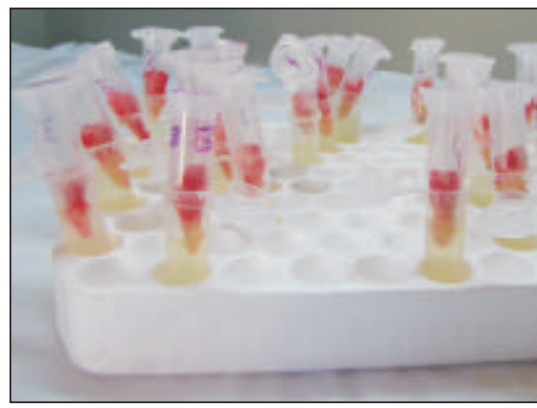

Fig 5: Apical 1/3 of teeth samples dipped in Enterococcus Faecalis broth.

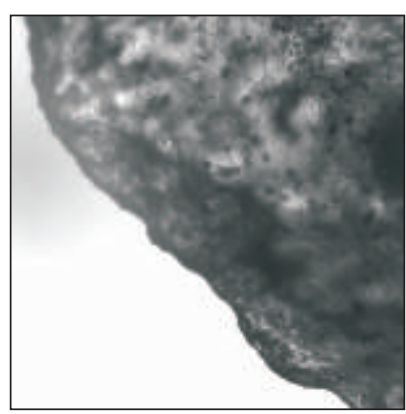

Fig 8: Group I samples showing absence of Enterococcus Faecalis.

Fig 6: inoculation chamber showing teeth samples placed in controlled chamber for bacterial growth.

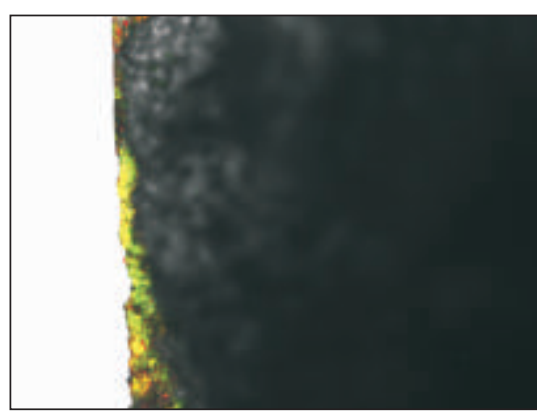

Fig 9: Group II samples showed presence of Enterococcus faecalis adhering to root cementum.

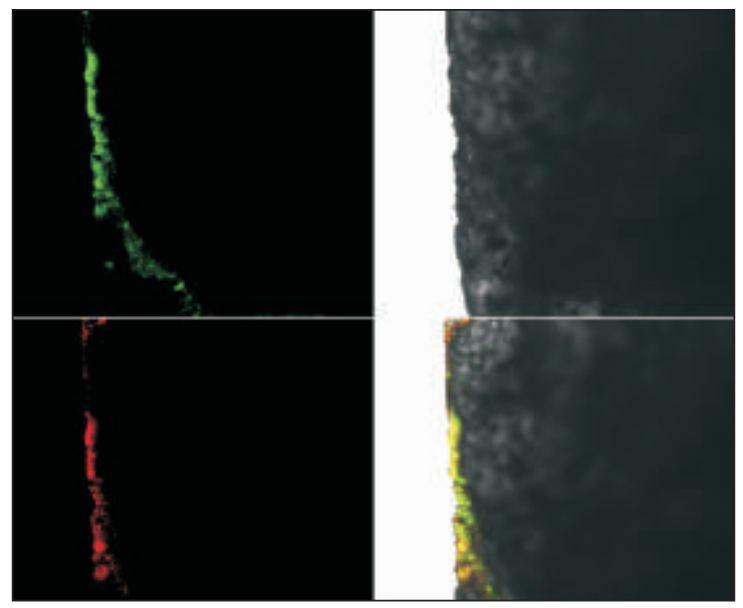

Fig 10: Separate picture showing live (green colour) and dead (red colour) Enterococcus Faecalis. 
Reference:

1. Haapasalo M , Ørstavik D. In vitro infection and disinfection of dentinal tubules. J Dent Res 1987;66:1375-1379.

2. Hubble TS, Hatton JF, Nallapareddy SR, Murray BE, Gillespie MJ. Influence of Enterococcus faecalis proteases and the collagen-binding protein, Ace, on adhesion to dentin. Oral Microbio Immun 2003;18:121-126.

3. Nallapareddy SR, Singh KV, Duh R-W, Weinstock GM, Murray BE. Diversity of ace, a gene encoding a microbial surface component recognizing adhesive matrix molecules, from different strains of Enterococcus faecalis and evidence for production of Ace during human infections. Infect Immun 2000a;68:5210-5217.

4. Nallapareddy SR, Qin X, Weinstock GM, Höök M, Murray BE. Enterococcus faecalis adhesin, Ace, mediates attachment to extracellular matrix proteins collagen type IV and laminin as well as collagen typel. Infect Immun 2000b;68:5218-5224.

5. Nallapareddy SR, Murray. BELigand-signaled upregulation of Enterococcus faecalis ace transcription, a mechanism for modulating host- E Faecalis interaction. Infect Immun 2006;74(9):4982-9.

6. Tandjung L, Waltimo T, Heide P, Decker EM, Weiger R. Octenidine in root canal and dentine disinfection EX vivo. Int Endod J 2007;40:84551.

7. Love RM. Enterococcus Faecalis- a mechanism for its role in endodontic failure. Int Endod J 2001;34:399-405.

8. Bergmans L, M oisiadis P, Huybrechts B, Van M eerbeek B, Quirynen M, Lambrechts P. Effect of photo-activated disinfection on endodontic pathogens ex vivo. Int Endod J 2008;41:227-39.

9. Ronald OZ, Ivaldo G de M, Thais HG, Roberto BG. Confocal laser scanning microscopy is appropriate to detect viability of enterococcus faecalisin dentin. Int Endod J 2008;34:1198-1201.

10. Zaura-Arite E, van M arie J, ten Cate JM. Confocal microscopy study of undisturbed and chlorhexidine-treated dental biofilm. J Dent Res 2001;80:1436-40.

11. Mason DJ, Lloyd D. Acridine orange as an indicator of bacterial susceptibility to gentamicin . FEM SM icrobiol Lett 1997;153:199-204.
12. Vitkov L, Hannig M, Krautgartner WD, et al. Ex vivo gingival biofilm consortia. Lett. Appl M icrobiol 2005;41:404-11.

13. Haapasalo M , Ørstavik D. In vitro infection and disinfection of dentinal tubules. J Dent Res 1987;66:1375-1379

14. M olander A, Reit C, Dahlén G, Kvist T. M icrobiological status of rootfilled teeth with apical periodontitis. Int EndodJ 1998;31:1-7.

15. Rich RL, Kreikemeyer B, Owens RT, LaBrenz S, Narayana SV, Weinstock $\mathrm{GM}$. Ace is a collagen-binding M SCRAM M from Enterococcus faecalis. J micro Chemis 1999; 274:26939-45

16. Evans M , Davies JK, Sundqvist G, Figdor D. M echanisms involved in the resistance of Enterococcus faecalis to calcium hydroxide. Int Endod J 2002;35:221-228.

17. Figdor D, Davies JK, Sundqvist G. Starvation survival, growth and recovery of Enterococcus faecalis in human serum. Oral Microbio Immun 2003;18:234-9.

18. Tronstad L, Andreasen JO, Hasselgren G, Kristerson L, Riis I. pH changes in dental tissues after root canal filling with calcium hydroxide. J Endod $1981 ; 7: 17-21$

19. Shungu DL, Cornett JB, Shockman GD. Morphological and physiological study of autolytic-defective Streptococcus faecium strains. J Bacteriol 1979;138:598-608.

20. Basinger SF, Jackson RW. Bacteriocin (hemolysin) of Streptococcus zymogenes. J Bacteriol 1968;96:1895-1902.

21. Jackson RW. Bacteriolysis and inhibition of Gram-positive bacteria by components of Streptococcus zymogenes lysine. I Bacteriol 1971;105:156-159

22. Jett BD, Gilmore MS. The growth-inhibitory effect of the Enterococcus faecalis bacteriocin encoded by pAD1 extends to the oral streptococci. Jent Res 1990;69: 1640-1645.

23. Tandjung I, Waltimo T, Hauser I, Heide P, Decker EM, Weiger R. Octenidine in root canal and dentine disinfection EX VIVO. Int Endod J 2007:40:845-51. 